\title{
DISTRIBUTIVE ELEMENTS IN THE NEAR-RINGS OF POLYNOMIALS
}

\author{
by JAIME GUTIERREZ* and CARLOS RUIZ DE VELASCO Y BELLAS
}

(Received 21st May 1987)

\section{Introduction}

As usual in the theory of polynomial near-rings, we deal with right near-rings. If $N=(N,+, \cdot)$ is a near-ring, the set of distributive elements of $N$ will be denoted by $N_{d}$;

$$
N_{d}=\{d \in N \mid d(r+s)=d r+d s, \text { for all } r, s \in N\} .
$$

It is easy to check that, if $N$ is an abelian near-ring (i.e., $r+s=s+r$, for all $r, s \in N$ ), then $N_{d}$ is a subring of $N$.

In this paper we describe the distributive elements of the near-ring of polynomials over a commutative ring with identity, which will be denoted by $R$. We also prove that if $R$ is an integral domain, the set of distributive elements contains the subrings of the near-ring of polynomials; in particular, the near-ring of polynomials has an unique maximal subring.

\section{The ring of the distributive elements}

The set $R[X]$, of all polynomials over $R$ in the indeterminate $X$, is a near-ring under addition "+" and substitution of polynomials "o" (i.e., $f(X) \circ g(X)=f(g(X))=f \circ g$ (see $[1]$, [4]). We shall denote by $R_{0}[X]$ the set of all polynomials over $R$ whose constant term is zero.

\subsection{Immediate properties}

(i) $R_{0}[X]$ is a subnear-ring of $(R[X],+, \circ)$ and agrees with $R[X]_{0}$, the zerosymmetric part of $R[X],+, \circ)$.

(ii) $\left(R[X]_{d},+, \circ\right)$ and $\left(R_{0}[X]_{d},+, \circ\right)$ are rings, and $R[X]_{d}$ is a subring of $R_{0}[X]_{d}$.

(iii) $\left(R[X]_{d},+\right)$ and $\left(R_{0}[X]_{d},+\right)$ are $R$-submodules of $R[X]$ and $R_{0}[X]$ (respectively).

*Partially supported by C.A.I.C. y T 2280/83. 
(iv) $\left(R[X]_{d},+, \circ\right)$ and $\left(R_{0}[X]_{d},+, \circ\right)$ are subrings of the ring $(\operatorname{End}(R[X]),+, \circ)$ and $\left(\operatorname{End}\left(R_{0}[X]\right),+, \circ\right)($ respectively).

Proof. (i) See [4, chap. 7-78].

(ii) The firsts two assertions are immediate. For the third, let $f \in R[X]_{d}$, let us say $f=a_{n} X^{n}+\cdots+a_{1} X+a_{0}$; then

$$
a_{0}=f \circ 0=f \circ(0+0)=f \circ 0+f \circ 0=a_{0}+a_{0} \text { hence } a_{0}=0 .
$$

(iii) They are immediate.

(iv) Consider the map $i: R[X]_{d} \rightarrow \operatorname{End}(R[X])$ defined by $i(f)(g)=f \circ g\left(f \in R[X]_{d}\right.$ and $g \in R[X])$; it is well defined and it is a morphism of rings.

Moreover, $\operatorname{Ker}(i)=\{0\}$. In fact, if $f \in \operatorname{Ker}(i)$, then $f=f \circ X=0$.

1.2. Consequence. The set $R X=\{a X \mid a \in R\}$ is a subring of $R[X]_{d}$ (resp. $\left.R_{0}[X]_{d}\right)$ isomorphic to $R$.

Proof. As $X \in R[X]_{d}$ and $R[X]_{d}$ is a $R$-module, this shows that $R[X]_{d} \supseteq R X$. Clearly $a X \circ b X=a b X$, and therefore $R X$ is a subring of $R[X]_{d}$.

Our main goal in this section is to find all the elements of $R[X]_{d}$ and $R_{0}[X]_{d}$. The proofs of several results are similar for both, so we shall just give the proof for either $R[X]_{d}$ or $R_{0}[X]_{d}$. First of all we reduce the problem to the case of monomials.

Lemma 1.3. Let a be a non-zero element of $R$ and let $n \geqq 2$, an integer:

(i) If $a X^{n} \notin R_{0}[X]_{d}\left(a X^{n} \notin R[X]_{d}\right)$, there exists an integer $i, 1 \leqq i \leqq n-1$, such that $a\left(\begin{array}{l}n \\ i\end{array}\right) \neq 0$ and for all $t \geqq 0, a X^{n} \circ\left(X^{t}+X^{t+1}\right) \neq a X^{n} \circ X^{t}+a X^{n} \circ X^{i+1}$.

(ii) If $a X^{n} \in R[X]_{d}\left(a X^{n} \in R_{0}[X]_{d}\right)$, then the order of a (denoted by $\left.0(a)\right)$ is finite.

Proof. (i) If $a X^{n} \notin R_{0}[X]_{d}$, there exists $f, g \in R_{0}[X]$ such that: $a X^{n} \circ(f+g) \neq a f^{n}+$ $a g^{n}$, hence for some $i, 1 \leqq i \leqq n-1$, we have $a\left(\begin{array}{l}n \\ i\end{array}\right) \neq 0$. Let $j=\max \left\{i / 1 \leqq i \leqq n-1, a\left(\begin{array}{l}n \\ i\end{array}\right) \neq 0\right\}$, then $a X^{n} \circ\left(X^{t}+X^{t+1}\right)=a\left(X^{t}(1+X)\right)^{n}=a X^{t n}(1+X)^{n}=a X^{t n}+a n X^{t n+1}+\cdots+a\left(\begin{array}{l}n \\ j\end{array}\right) X^{t n+j}+$ $a X^{i n} X^{n}$, and $a\left(\begin{array}{l}n \\ j\end{array}\right) X^{i n+j} \neq 0$.

(ii) If $O(a)$ is infinite, we have

$$
\begin{aligned}
a X^{n} \circ\left(X+X^{2}\right) & =a X^{n}+a n X^{n+1}+\cdots+a n X^{2 n-1}+a X^{2 n} \neq a X^{n} \circ X+a X^{n} \circ X^{2} \\
& =a X^{n}+a X^{2 n}
\end{aligned}
$$

by hypothesis $a n X^{2 n-1} \neq 0$, which leads to a contradiction. 
Proposition 1.4. Let $f=a_{n} X^{n}+\cdots+a_{1} X \in R[X]$; then $f \in R[X]_{d}$ (resp. $R_{0}[X]_{d}$ ) if and only if $a_{i} X^{i} \in R[X]_{d}\left(\right.$ resp. $\left.R_{0}[X]_{d}\right)$ for all $i=1, \ldots, n$.

Proof. Suppose $f \in R[X]_{d}$ and $a_{n} X^{n} \notin R[X]_{d}$, and we consider, $j=\max \{i / 1 \leqq i \leqq n-1$, $\left.a_{n}\left(\begin{array}{l}n \\ i\end{array}\right) \neq 0\right\}$ (see Lemma 1.3) and $t$ an integer $\geqq 1$; then we get $f(X) \circ\left(X^{t}+X^{t+1}\right)=$ $f\left(X^{t}\right)+f\left(X^{t+1}\right)=a_{n} X^{t n}+\cdots+a_{1} X^{t}+a_{n} X^{(t+1) n}+\cdots+a_{1} X^{t+1} \equiv(*)$. On the other hand $f(X) \circ\left(X^{i}+X^{i+1}\right)=a_{n}\left(X^{i}+X^{i+1}\right)^{n}+\cdots+a_{1}\left(X^{i}+X^{t+1}\right) \equiv(* *)$. Moreover, the first summand of (**) is: $a_{n}\left(X^{i}+X^{t+1}\right)^{n}=a_{n} X^{t n}+\cdots+a_{n}\left(\begin{array}{l}n \\ j\end{array}\right) X^{i n+j}+a_{n} X^{(t+1) n}$, (with $a_{n}\left(\begin{array}{l}n \\ j\end{array}\right) X^{i n+j} \neq 0$ which is the highest degree monomial (different from $a_{n} X^{(t+1) n}$ ) occurring in the development of $a_{n}\left(X^{t}+X^{t+1}\right)^{n}$. We now prove that for a large enough integer $t$, $a_{n}\left(\begin{array}{l}n \\ j\end{array}\right) X^{t^{n+j}} \neq 0$ is the highest degree monomial (different from $a_{n} X^{(i+1) n}$ ) occurring in the development of the polynomial $f(X) \circ\left(X^{t}+X^{t+1}\right)$. In fact, the monomials of $f(X)$ 。 $\left(X^{t}+X^{t+1}\right)$ are all of the form $a_{m}\left(\begin{array}{l}m \\ k\end{array}\right) X^{t m+k}$ with $0 \leqq k \leqq m<n$ except for the monomials given by $a_{n} X^{n}$ (a case already studied above). Now, we can choose an integer $t$ large enough such that $t n+j>t m+k$; since $(*)=(* *)$, contradiction.

Theorem 1.5. If all the non-zero elements of $R$ have infinite order (torsion free), then: $R[X]_{d}=R_{0}[X]_{d}=R X$.

Proof. It is an immediate consequence of 1.1(ii), 1.3(ii) and 1.4.

We now prove some preliminary lemmas for the explicit description of $R[X]_{d}$ and $R_{0}[X]_{d}$ in the remaining cases.

Lemma 1.6. Let $n, p$ be two integers such that $n \geqq 1$ and $p$ is a prime number. Suppose $n=p^{a} r$, where $a$ is a non-negative integer and $r$ is a positive integer such that $p$ does not divide $r$. Then, if $t \leqq a$, the integer $\left(\begin{array}{l}n \\ p^{2}\end{array}\right)$ is divisible by $p^{a-t}$ but it is not divisible by $p^{a-t+1}$.

From this last result it is easy to prove the following lemma, of which we have not found any references in the literature.

Lemma 1.7. Let $n>1$ be an integer. The greatest common divisor (gcd) of $\left\{\left(\begin{array}{l}n \\ i\end{array}\right) \mid i=1,2, \ldots, n-1\right\}$ is $p$ if $n$ is a power of a prime number $p$, and 1 otherwise.

Proof. Let $d$ be the gcd of $\left\{\left(\begin{array}{c}n \\ i\end{array}\right) \mid i=1,2, \ldots, n-1\right\}$. If $n$ is a power of the prime $p$, say $n=p^{a}$, then $d$ divides $\left(\begin{array}{l}n \\ p^{a-1}\end{array}\right)$, and by Lemma 1.6 we get $d=p$. Now, if $n=p_{1}^{a_{1}} \ldots p_{t}^{a_{1}}$, with $t \geqq 2$, then $d=p_{1}^{\alpha_{1}} \ldots p_{t}^{\alpha_{t}}$ with $0 \leqq \alpha_{i} \leqq a_{i}$; as $d$ divides $\left(\begin{array}{c}n_{i} \alpha_{i} \\ )\end{array}\right)$ for all $i, i=1, \ldots, t$, using again the lemma, we conclude $\alpha_{i}=0, i=1, \ldots, t$.

Proposition 1.8. Let $a \neq 0$ be an element of $R$ and let $n \geqq 2$ be an integer. Then $a X^{n} \in R[X]_{d}\left(\right.$ resp. $\left.R_{0}[X]_{d}\right)$ if and only if the order of a divides $\left(\begin{array}{l}n \\ i\end{array}\right)$ for all $i=1, \ldots, n-1$.

Proof. Let us suppose $a X^{n} \in R[X]_{d}$, we have $a X^{n}(1+X)^{n}=a(X(1+X))^{n}=a(X+$ 
$\left.X^{2}\right)^{n}=a X^{n} \circ\left(X+X^{2}\right)=a X^{n}+a X^{2 n}$. By expanding the first member, we notice $a\left(\begin{array}{l}n \\ i\end{array}\right) X^{n+i}=$ 0 for all $i=1,2, \ldots, n-1$; hence $a\left(\begin{array}{l}n \\ i\end{array}\right)=0$.

Theorem 1.9. Let $a \neq 0$ be an element of $R$, and let $n \geqq 2$ be an integer; then $a X^{n} \in R[X]_{d}$ (resp. $\left.R_{0}[X]_{d}\right)$ if and only if there exists a prime $p$ and a positive integer $\alpha$ such that $n=p^{\alpha}$ and $0(a)=p$.

Proof. Let us assume $a X^{n} \in R[X]_{d}$, by Proposition $1.8 O(a)$ divides $\operatorname{gcd}\left\{\left(\begin{array}{l}n \\ i\end{array}\right) \mid i=\right.$ $1, \ldots, n-1\}$; as $0(a)>1$, using Lemma 1.7 , we have $n=p^{\alpha}$ for some prime $p$ and an integer $\alpha>0$. Now the converse is obvious.

As a consequence of 1.4 and 1.9 we obtain the following:

Theorem 1.10. $R[X]_{d}=R_{0}[X]_{d}$.

In order to get an explicit description of the ring $R[X]_{d}$, we introduce the following notation: given a prime $p$

$$
\begin{aligned}
\mathbf{I}_{p}: & =\{a \in R \mid 0(a)=p\} \cup\{0\}, \\
\mathbf{I}_{p}[X]: & =\left\{a_{n} X^{p^{n}}+a_{n-1} X^{p^{n-1}}+\cdots+a_{1} X^{p^{1}} / a_{i} \in \mathbf{I}_{p}, n \geqq 1\right\} .
\end{aligned}
$$

Lemma 1.11. (i) For every prime $p, I_{p}$ is an ideal of $R$.

(ii) If $p, q$ are different primes, the set $\mathbf{I}_{p} \mathbf{I}_{q}:=\left\{a b \mid a \in \mathbf{I}_{p}, b \in \mathbf{I}_{q}\right\}=\{0\}$.

(iii) For every prime $p, \mathbf{I}_{p}[X]$ is an ideal of $R[X]_{d}$.

Proof. It is straightforward; for (iii) it is enough to consider monomials and use (i) and (ii).

Theorem 1.12. We have

$$
R[X]_{d}=\left(\bigoplus_{p \in p} I_{p}[X]\right) \oplus R X
$$

where $\mathbf{P}$ denotes the set of all prime numbers.

Proof. It is an immediate consequence of 1.9 and 1.11 .

We note that $R X$ is a subring of $R[X]_{d}$, but it is not an ideal. In the following corollary we express $R[X]_{d}$ as a direct sum of ideals in some particular cases. For every prime $p$ we define 


$$
\mathbf{I}_{p}^{*}[X]:=\left\{a_{n} X^{p^{n}}+a_{n-1} X^{p^{n-1}}+\cdots+a_{1} X^{p^{1}}+a_{0} X^{p^{0}} / a_{i} \in \mathbf{I}_{p}, n \geqq 0\right\}
$$

Lemma 1.13. For every prime $p, I_{p}^{*}[X]$ is an ideal of $R[X]_{d}$.

Corollary 1.14. Let $R$ be a unitary commutative ring with the following property $(P)$ : "there are some prime numbers $p_{1}, \ldots, p_{s}$ and elements $a_{1}$ in $\mathbf{I}_{p 1}, \ldots, a_{s}$ in $\mathbf{I}_{p_{s}}$ such that $1=a_{1}+\cdots+a_{s}$, then:

$$
R[X]_{d}=\bigoplus_{p \in P} I_{p}^{*}[X]
$$

The property required in Corollary 1.14 is not always verified as we can see in the following:

Examples 1.15. (i) Let $R$ be a ring of characteristic an integer $n>1$ such that $n=p_{1} p_{2} \ldots p_{r}, \quad r>1$ and $p_{1}, p_{2}, \ldots, p_{r}$ distinct primes; we have here $p_{1} \ldots p_{i-1} \hat{p}_{i} p_{i+1} \ldots p_{r} \in \mathbf{I}_{p i}$, for all $i=1,2, \ldots, r$; where "denotes omission of the $p_{i}$. As

$$
\operatorname{gcd}\left(p_{2} \ldots p_{r} \ldots p_{1} \ldots p_{i-1} p_{i+1} \ldots p_{r} \ldots p_{1} p_{2} \ldots p_{r-1}\right)=1
$$

we get $1=a_{1}+\cdots+a_{r}$ with $a_{i} \in \mathbf{I}_{p_{i}}$, so this kind (or class) of rings verifies the above property $(P)$.

(ii) Let $R=Z_{12}$, the integers modulo 12 , then $\mathbf{I}_{2}=\{0,6\}, \mathbf{I}_{3}=\{0,4,8\}$ and $\mathbf{I}_{p}=\{0\}$ otherwise. In this case 1 cannot be expressed as a sum of elements of the $I_{p}$ 's, hence this ring does not verify the property.

Corollary 1.16. If the characteristic of $R$ is a prime number $p$, then

$$
R[X]_{d}=\left\{a_{n} X^{p^{n}}+a_{n-1} X^{p^{n-1}}+\cdots+a_{1} X^{p^{1}}+a_{0} X^{p^{0}} / a_{i} \in R, n \geqq 0\right\} .
$$

The elements of $R[X]_{d}$ are, in this case, the so called $p$-polynomials. They were introduced and studied by Ore when $R$ is a finite field, but in another context, they have interesting properties (see [3]). See also [2] pages 108 onwards and references there mentioned.

\section{Rings in near-rings of polynomials}

In this section, we investigate rings which are contained in $R[X]$. Since all rings are zero-symmetric near-rings, we only need to search for them in $R_{0}[X]$.

We prove our main result:

Theorem 2.1. Let $S$ be a subring of $R[X]$ (not necessarily unitary). If $R$ is an integral domain then $S$ is contained in $R[X]_{d}$. 
The proof requires a series of lemmas as well as a number of results from Section 1.

Lemma 2.2. Let $R$ be an integral domain and let $S$ be a subring of $R[X]$ (not necessarily unitary) then: $f \circ(X+f)=f+f \circ f$, for all $f \in S$.

Proof. Let $f \neq 0$ then $f, f \circ f \neq 0 \in S$, we have $f \circ(f+f \circ f)=f \circ f+f \circ f \circ f=(f+$ $f \circ f) \circ f$, but on the other hand $f \circ(f+f \circ f)=f \circ(X+f) \circ f$, since $R$ is an integral domain hence $f$ is right cancellable (see [1]).

The characteristic of an integral domain is either 0 or a prime number $p$. We treat those cases separately and start with:

Proposition 2.3. Let $R$ be an integral domain of characteristic 0 and let $S$ be a subring of $R[X]$ (not necessarily unitary) then: $S$ is contained in $R[X]_{d}$.

Proof. Let $f=a_{n} X^{n}+\cdots a_{1} X \in S$, by the last lemma; $f \circ(X+f)=f+f \circ f$, then $a_{n}(X+f)^{n}+\cdots+a_{1}(X+f)=a_{n} X^{n}+\cdots+a_{1} X+a_{n} f^{n}+\cdots+a_{1} f$, we get $n=1$ or $a_{n}=0$, and we end the proof using Proposition 1.5.

Corollary 2.4. Let $R$ be an-integral domain of characteristic 0 then the subrings of $R[X]$ are (isomorphic to) subrings of the ring $R$.

Proof. It is immediate using $1.2,1.5$ and 2.3 .

Hence we have proved our Theorem 2.1 in the case when the characteristic of $R$ is 0 . Now we consider the case of characteristic a prime number $p$.

Lemma 2.5. (a) The set $R^{\prime}[X]_{0}:=\left\{f \in R_{0}[x] / f^{\prime}=\right.$ constant, where $f^{\prime}$ is the formal derivative of $f\}$ is a subnear-ring of $R[X]$ containing $R[X]_{d}$ and moreover $R^{\prime}[X]_{0}=$ $R[X]_{d}$ if and only if $R$ is torsion free.

(b) If $R$ is an integral domain of characteristic a prime number p. Let $a \neq 0$ be an element of $R$ and let $n \geqq 2$ be an integer then: $a X^{n} \in R^{\prime}[X]_{0}$ if and only if $p$ divides $n$.

Proof. (a) The first assertion is straightforward. It suffices to observe that $f=$ $a_{n} X^{n}+\cdots+a_{1} X \in R^{\prime}[X]_{0}$ if and only if $a_{i} X^{i} \in R^{\prime}[X]_{0}$, for all $i=1$ to $n$, now use 1.9. For the second assertion; let $a \neq 0$ be an element of $R$ and let $n \geqq 2$ be an integer such that $n a=0$ and $n=p_{1}^{r_{1}} \ldots p_{t}^{r_{2}}$. We distinguish two cases: if $t \geqq 2$, then $a X^{n} \in R^{\prime}[X]_{0}$ but

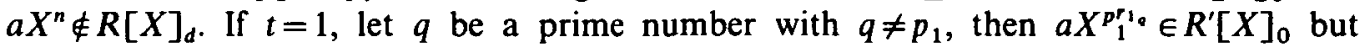
$a X_{1}^{p_{1}{ }_{1}} \notin R[X]_{d}$. The converse is immediate.

(b) is immediate.

Lemma 2.6. Let $R$ be an integral domain of characteristic a prime number $p$ and let $S$ be a subring of $R[X]$ (not necessarily unitary) then: $S$ is contained in $R^{\prime}[X]_{0}$. 
Proof. Let $f=a_{n} X^{n}+\cdots+a_{1} X \in S$. If $n=1$ then $S$ is contained in $R^{\prime}[X]_{0}$. Suppose $n \geqq 2$. First we show that $p$ divides $n$. Suppose $\operatorname{gcd}(n, p)=1$ by Lemma $2.2 f \circ(X+$ $f)=f+f \circ f$, we have $\left(a_{n} X^{n}+\cdots+a_{1} X\right) \circ(X+f)=a_{n}(X+f)^{n}+\cdots+a_{1}(X+f)$, the first summand $a_{n}(X+f)^{n}=a_{n} X^{n}+\cdots+n a_{n} f^{n-1} X+a_{n} f^{n}$; we see $n a_{n} f^{n-1} X=n a_{n}^{n} X^{n(n-1)+1}+$ $\ldots$, with $n a_{n}^{n} X^{n(n-1)+1} \neq 0$, so $f \circ(X+f)-(f+f \circ f)=n a_{n}^{n} X^{n(n-1)+1}+\ldots \neq 0$, contradiction. Hence $p$ divides $n$.

We take $r=\max \left\{i / i=1,2, \ldots, n, a_{i} \neq 0\right.$ and $\left.\operatorname{gcd}(i, p)=1\right\}$. Two cases occur:

(i) If $r=1$ by $2.5 f \in R^{\prime}[X]_{0}$, then $S$ is contained in $R^{\prime}[\mathrm{X}]_{0}$.

(ii) If $r \geqq 2$, we have $f=a_{n} X^{n}+\cdots+a_{r} X^{r}+\cdots+a_{1} X$ with $a_{r} \neq 0, r<n, g c d(r, p)=1$ again by $2.2 f \circ(X+f)=f+f \circ f$ and by the properties of the derivative we get: $\left(f^{\prime} \circ(X+f)\right)\left(1+f^{\prime}\right)=f^{\prime}+\left(f^{\prime} \circ f\right) f^{\prime}$ on the right hand side we have $\left(f^{\prime} \circ(X+\right.$ $f))\left(1+f^{\prime}\right)=\left(\left(r a_{r} X^{r-1}+\cdots+a_{1}\right) \circ(X+f)\right)\left(1+f^{\prime}\right)=\left(r a_{r}(X+f)^{r-1}+\cdots+a_{1}\right)+$ $\left(r a_{r}(X+f)^{r-1}+\cdots+a_{1}\right) f^{\prime}$. Let $g=\left(r a_{r}(X+f)^{r-1}+\cdots+a_{1}\right)-f^{\prime}$, then $g \neq 0$ and the degree of $g$ is $n(r-1)$. Let $h=\left(r a_{r}(X+f)^{r-1}+\cdots+a_{1}\right) f^{\prime}-\left(f^{\prime} \circ f\right) f^{\prime}$, then the degree of $h, t$ is $t<n(r-1)$. So $\left(f^{\prime} \circ(X+f)\right)\left(1+f^{\prime}\right)-f^{\prime}+\left(f^{\prime} \circ f\right) f^{\prime}=$ $g(X)+h(X) \neq 0$, a contradiction. Therefore $r=1$ and $f \in R^{\prime}[X]_{0}$.

Proposition 2.7. Let $R$ be an integral domain of characteristic a prime number $p$ and let $S$ be a subring of $R[X]$ (not neccessarily unitary) then: $S$ is contained in $R[X]_{d}$.

Proof. Let $f=a_{n} X^{n}+\cdots+a_{1} X \in S$. There exist $h, g \in R[X]$ such that $f=h+g$, with $g \in R[X]_{d}$ and $h=b_{m} X^{m}+\cdots+b_{t} X^{t}$, with $b_{i} X^{i} \notin R[X]_{d}$ for all $i=1, \ldots, m$ and $t>1$. If $h=0$, then $f \in R[X]_{d}$ and $S$ is contained in $R[X]_{d}$.

Suppose $h \neq 0$, by Lemma $2.6 f \in R^{\prime}[X]_{0}$ so $h \in R^{\prime}[X]_{0}$. By Lemma 2.5 we get

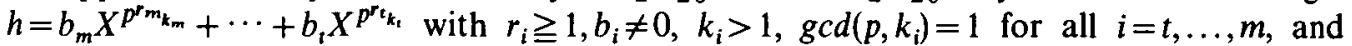
$p^{r_{j}} k_{j}>p^{r_{i}} k_{i}$ for all $j>i$.

Let $r_{h}=\min \left\{r_{i} / i=t, \ldots, m\right\}$. To simplify notation, we shall write $k_{h}=k$ and $r_{h}=r$; then $h=\left(b_{m} X^{p^{s m_{k_{m}}}}+\cdots+b_{h} X^{k}+\cdots+b_{t} X^{p^{s_{k_{k}}}}\right) \circ X^{p^{r}}$. Let $F$ be the quotient field of $R$ and let $\bar{F}$

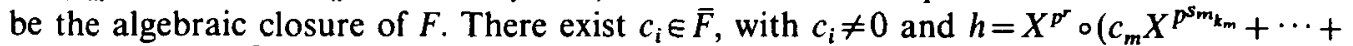
$\left.c_{h} X^{k}+\cdots+c_{t} X^{p^{s_{k_{t}}}}\right)$. We can write $h(X)=X^{p^{r}} \circ c$, where $c=c_{m} X^{p^{s_{m_{m}}}}+\cdots+c_{h} X^{k}+\cdots+$

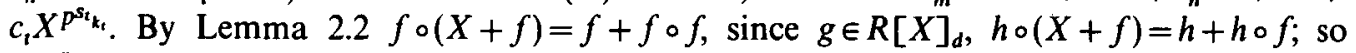
$\left(X^{p^{r}} \circ c\right) \circ(X+f)=X^{p^{r}} \circ c+X^{p^{r}} \circ c \circ f$, as $X^{p^{r}} \in F[X]_{d}$ we have $X^{p^{r}} \circ(c \circ(X+f))=X^{p^{r}} \circ(c+$ $c \circ f)$, since $r>1$ and since $R$ is an integral domain then $c \circ(X+f)=c+c \circ f$. Using the properties of the derivative we have $\left(c^{\prime} \circ(X+f)\right)\left(1+f^{\prime}\right)=c^{\prime}+\left(c^{\prime} \circ f\right) f^{\prime}$ and we arrive at a contradiction. The proof is similar to the one in Lemma 2.6 and is therefore omitted.

This completes the proof of Theorem 2.1.

Remark 2.8. If $R$ is not an integral domain, then Theorem 2.1 does not hold: we take $R=Z_{4}$, the ring of integers modulo 4 . Let $B$ be the group generated by $\left\langle X, 2 X^{3 i}\right.$ for 
all $i \geqq 0\rangle$ then $B=\left\langle X, 2 X^{3^{i}}\right.$ for all $\left.i \geqq 0\right\rangle$ is an infinite unitary ring, but $B$ is not contained in $R[X]_{d}$. We also see that $B$ is not contained in $R^{\prime}[X]_{0}$.

Corollary 2.9. Let $R$ be an integral domain of characteristic a prime number $p$, then:

(i) $R[X]$ has an unique maximal subring.

(ii) $R[X]$ has a subring $S$ isomorphic to the polynomial ring $\left(Z_{p}[X],+, \cdot\right)$, where $Z_{p}$ is the field of integers modulo $p$. In particular, the subrings of $Z_{p}[X]$ are (isomorphic to) subrings of the polynomial ring $\left(Z_{p}[X],+, \cdot\right)$.

Proof. (ii) The map $\phi$ from $\left(Z_{p}[X]_{d},+, \circ\right)$ to $\left(Z_{p}[X],+, \cdot\right)$, defined as follows: $\phi\left(a X^{p^{n}}\right)=a X^{n}$ is a ring isomorphism. The proof is now immediate using 1.16 and 2.7.

Acknowledgement. We would like to thank Professor Günter Pilz for his generous help.

\section{REFERENCES}

1. H. LausCh and W. NÖbauer, Algebra of polynomials, (North-Holland, Amsterdam-New York, 1973).

2. R. LidL and H. NeIDerReiter, Finite fields (Addison-Wesley, Reading, Massachusetts, 1983).

3. O. Ore, On a special class of polynomials, Trans. Amer. Math. Soc. 35 (1933), 539-584.

4. G. PILz, Near-rings (North-Holland, Amsterdam, 1983).

Departmento de Matematicas

Facultad de Ciencias

UNIVERSIDAD DE CANTABRIA

39005-S ANTANDER, Spain. 